\title{
Symmetrical Acyclic Aryl Aldazines with Antibacterial and Antifungal Activity
}

\author{
Vanya B. Kurteva, ${ }^{1, *}$ Svilen P. Simeonov, ${ }^{1}$ Margarita Stoilova-Disheva ${ }^{2}$ \\ ${ }^{1}$ Institute of Organic Chemistry with Centre of Phytochemistry, Bulgarian Academy of Sciences, Sofia, Bulgaria; ${ }^{2}$ Institute of Micro- \\ biology, Bulgarian Academy of Sciences, Sofia, Bulgaria. \\ Email: vkurteva@orgchm.bas.bg
}

Received October $19^{\text {th }}, 2010$; revised November $8^{\text {th }}, 2010$; accepted November $15^{\text {th }}, 2010$.

\begin{abstract}
A series of 22 symmetrical acyclic aromatic aldazines were obtained and their qualitative antimicrobial activities were evaluated against 10 bacterial and 3 fungal species. The results demonstrated that the bi- and polycyclic aromatics studied are remarkably more active than benzaldazines. The latter possess antibacterial activities only, which were dramatically reduced by the introduction of substituents. The tests showed that the activities are strongly dependent on the type and position of the substituents and that the effects on antibacterial and antifungal activities are the opposite. 2-Naphtaldazine was significantly more active than its position isomer 1-naphthaldazine against Saccharomyces cerevisiae and Penicillium chrysogenum, whereas both compounds possess commensurable activities towards Candida tropicalis and the bacterial strains. From the other side, the presence of 4-hydroxy substituent in 1-naphthaldazine reduced the antibacterial and increased the antifungal activities, while the influence of 2-hydroxy group led to reversed results.
\end{abstract}

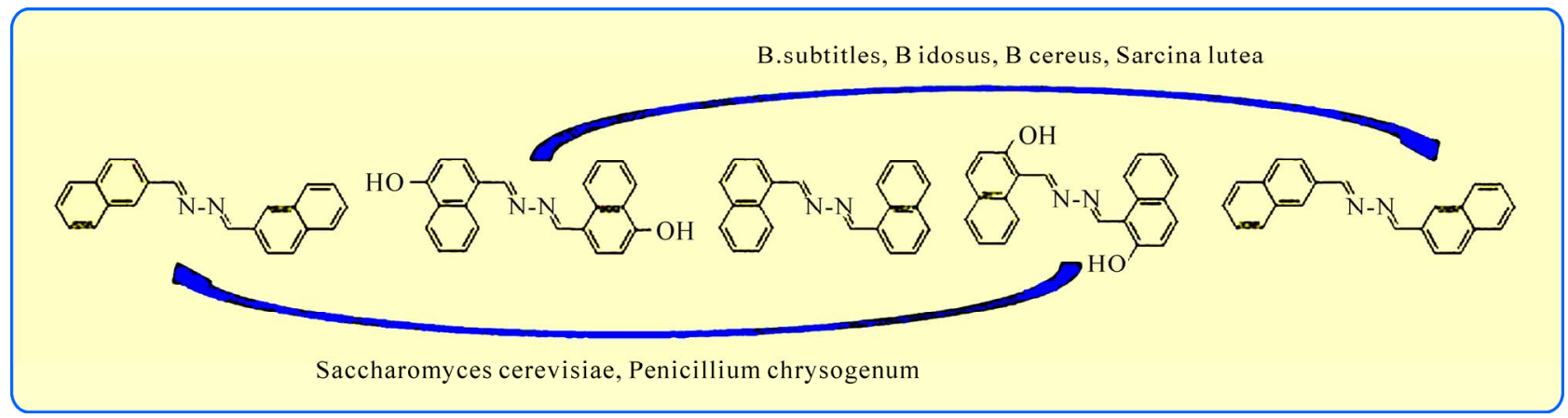

Keywords: Symmetrical Acyclic Aryl Aldazines, Synthesis, Antibacterial Activity, Antifungal Activity

\section{Introduction}

Azines are a class of organic compounds containing $\mathrm{R}_{1} \mathrm{R}_{2} \mathrm{C}=\mathrm{N}-\mathrm{N}=\mathrm{CR}_{3} \mathrm{R}_{4}$ fragment. Depending on the nature of the substituents $\mathrm{R}$, they are divided in several sub-groups: 1$)$ symmetrical $\left(\mathrm{R}_{1} \mathrm{R}_{2}=\mathrm{R}_{3} \mathrm{R}_{4}\right)$ and unsymmetrical $\left.\left(R_{1} R_{2} \neq R_{3} R_{4}\right) ; 2\right)$ aldazines $\left(R_{1}=R_{3}=H\right)$, ketazines $\left(R_{1} R_{2} R_{3} R_{4} \neq H\right)$ and mixed azines $\left(R_{1}=H, R_{2} R_{3} R_{4} \neq\right.$ $\mathrm{H})$; 3) aromatic and aliphatic; 4) cyclic and acyclic. Numerous examples are well-known ever since $19^{\text {th }}$ century but only a limited number of synthetic pathways are applied for their preparation. The classical scheme for the construction of acyclic aldazines is based on a reaction of aldehydes with hydrazine [1,2]. Despite the high toxicity of the latter, the protocol is very fast, simple and efficient and is still widely exploited. Semicarbazide has been also applied as a reagent in a two-step procedure involving thermolysis of the intermediately formed semicarbazones at high temperature [3,4]. Recently, the transformation has achieved as an environmentally benign solvent-free procedure under microwave irradiation $[5,6]$.

Compounds possessing azine moiety are still the order of the day due to the broad spectrum of biological activeity profiles displayed. Ketazines, mixed azines, and cyclic compounds have exhibited antitumor [7-12], anti- 
bacterial [13-20], anti-inflammatory [21], antimalarial dyes [22], anticonvulsant $[23,24]$, insect growth regulators [25] and many other activities. Contrary, acyclic aldazines are much less studied. Unsymmetrical aldazines have shown antitumor [26], antibacterial [27,28], and antioxidant [29] activity. Symmetrical 4-bromo benzaldazine has evaluated as anticonvulsant agent but shown very low activity [30]. Similar monosubstituted benzaldazines have been tested as allosteric modulators [31] and has found that 2-fluoro and 3-fluoro compounds possessed positive activity, while 4-fluoro, 3-chloro, 3and 4-methoxy, and 3-hydroxy analogues were not active. Recently, a series of unsymmetrical and two examples of symmetrical 3-indolyl aldazines have been studied and have found to exhibit antioxidant [29] and antibacterial [28] activity. The latter presents, to the best of our knowledge, the only record in the literature on the evaluation of antibacterial and antifungal activity of symmetrical aryl aldazines.

In this paper, we report on a synthesis, characterization and qualitative antibacterial and antifungal activity evaluation of a series of 22 symmetrical acyclic aryl aldazines.

\section{Results and Discussion}

\subsection{Chemistry}

The titled symmetrical aldazines were obtained by the classical protocol from aldehydes and hydrazine sulphate in ethanol, as shown on Scheme 1. Aromatic aldehydes with varied ring size and type and position of the substituents were chosen in an attempt to study the influence of different factors on the activity. The syntheses of aldazines 20-22 were carried out in ethanol/dimethylformamide instead of pure ethanol due to the limited solubility of the starting aldehydes. The compounds 1-16 and
18-22 were isolated in high to excellent yields (Table 1) after a very simple work-up. The E,E-configuration of the products was assumed on the bases of the previously confirmed by X-ray analysis stereochemistry of a similar symmetrical aryl aldazine sample [6].

The naphthaldazine 17 possessing a non-conjugated side-chain was obtained in moderate yield from 15 by Mannich reaction (Scheme 2, Table 1). As it was found that 17 is less active than the corresponding unsubstituted compound 15, other examples were not synthesized.

All known aldazines, 1-11, 13-16, 18, 19, 21, and 22, were characterized by a comparison of the melting points with the literature data (Table 1) and by their NMR spectra, given in the experimental section. The structures of the new members, 12, 17 and 20, were additionally confirmed by electrospray ionization mass spectrometry.

\subsection{Pharmacology}

The in vitro antibacterial and antifungal activities of the synthesized compounds against a series of species were determined qualitatively by the agar cup test according to the European Pharmacopoeia [48]. Shortly, suspensions of the test microorganisms were inoculated into sterile melted nutrient agar media and poured into Petri dishes. The bacterial strains were grown in nutrient agar (Serva, Germany) for $24 \mathrm{~h}$ at $37^{\circ} \mathrm{C}$ while the yeast and fungal strains were incubated in yeast peptone dextrose agar (YEPD) and in potato dextrose agar (PDA), respectively for $72 \mathrm{~h}$ at $28^{\circ} \mathrm{C}$. Six per dish wells, each $8 \mathrm{~mm}$ in diameter, were prepared. Fifty microliter of each sample in dimethylsulfoxide $(25 \mathrm{mg} / \mathrm{ml}$ or $12.5 \mathrm{mg} / \mathrm{ml})$ was added to the appropriate well. For pre-diffision the Petri dishes were placed at $4^{\circ} \mathrm{C}$ for $2 \mathrm{~h}$. The antimicrobial activity was estimated by the diameter of inhibitory zones $(\mathrm{mm})$ in the agar layer. Control experiments were carried out with the pure solvent.

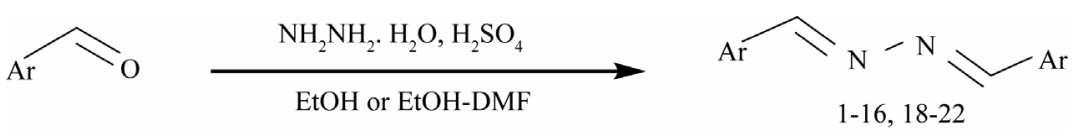

Scheme 1. Synthesis of symmetrical aryl aldazines 1-16 and 18-22.
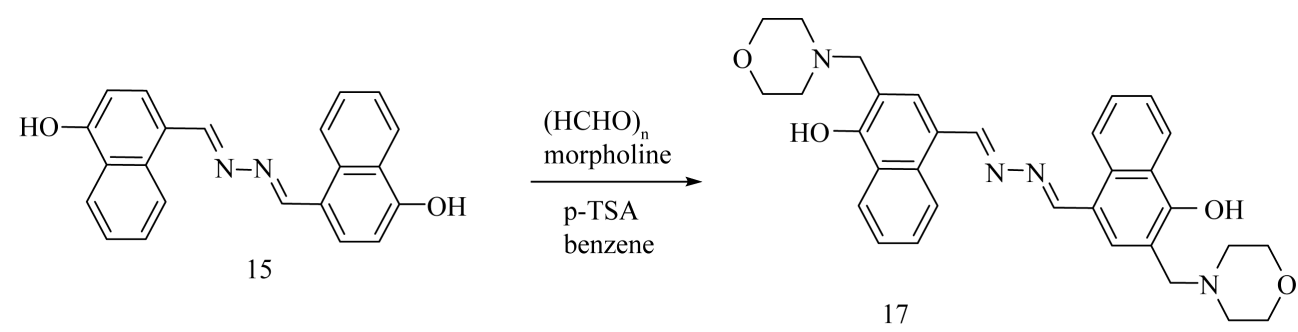

Scheme 2. Synthesis of symmetrical aryl naphthaldazine 17. 
Table 1. Synthesis and physical data of symmetrical aryl aldazines 1-22.

\begin{tabular}{|c|c|c|c|c|}
\hline Azine & Ar group & Yield, \% & m.p., ${ }^{\circ} \mathrm{C}$ (lit.) & $\mathrm{R}_{\mathrm{f}}$-value, $(\mathrm{MP})^{\mathrm{a}}$ \\
\hline 1 & $\mathrm{Ph}$ & 90 & $91.5-92(92[32])$ & $0.63(\mathrm{~A})$ \\
\hline 2 & $4-\mathrm{OH}-\mathrm{C}_{6} \mathrm{H}_{4}$ & 88 & $258-259(268[33])$ & $0.36(\mathrm{~B})$ \\
\hline 3 & $3-\mathrm{OH}-\mathrm{C}_{6} \mathrm{H}_{4}$ & 94 & $205.5-206(205$ [34]) & $0.51(\mathrm{~B})$ \\
\hline 4 & $2-\mathrm{OH}-\mathrm{C}_{6} \mathrm{H}_{4}$ & 96 & $210-211(213-214[3])$ & $0.85(\mathrm{~B})$ \\
\hline 5 & 3-OMe-2-OH- $\mathrm{C}_{6} \mathrm{H}_{3}$ & 98 & $192-193(196[35])$ & 0.47 (A) \\
\hline 6 & $4-\mathrm{NMe}_{2}-\mathrm{C}_{6} \mathrm{H}_{4}$ & 87 & $254.5-255(250-253[33])$ & $0.59(\mathrm{~A})$ \\
\hline 7 & $4-\mathrm{Cl}-\mathrm{C}_{6} \mathrm{H}_{4}$ & 93 & $210-211(213[36])$ & $0.71(\mathrm{~A})$ \\
\hline 8 & $3-\mathrm{Cl}-\mathrm{C}_{6} \mathrm{H}_{4}$ & 93 & $142-143(141[37])$ & $0.75(\mathrm{~A})$ \\
\hline 9 & $2-\mathrm{Cl}-\mathrm{C}_{6} \mathrm{H}_{4}$ & 89 & $143.5-144(143.5[38])$ & 0.77 (A) \\
\hline 10 & $2,6-\mathrm{Cl}_{2}-\mathrm{C}_{6} \mathrm{H}_{3}$ & 87 & $152.5-153(153$ [39]) & $0.76(\mathrm{~A})$ \\
\hline 11 & $2,4-\mathrm{Cl}_{2}-\mathrm{C}_{6} \mathrm{H}_{3}$ & 95 & $218-219(213[40])$ & $0.83(\mathrm{~A})$ \\
\hline 12 & $2-\mathrm{Cl}-4-\mathrm{F}-\mathrm{C}_{6} \mathrm{H}_{3}$ & 97 & $193.5-194$ & $0.80(\mathrm{~A})$ \\
\hline 13 & $3-\mathrm{F}-\mathrm{C}_{6} \mathrm{H}_{4}$ & 91 & $134-135(132$ [41]) & $0.72(\mathrm{~A})$ \\
\hline 14 & 1-naphthyl & 96 & $156-156.5(156[42])$ & $0.74(\mathrm{~A})$ \\
\hline 15 & 4-OH-1-naphthyl & 84 & $235-236(236[43])$ & 0.58 (B) \\
\hline 16 & 2-OH-1-naphthyl & 89 & $290-293(>290[44])$ & 0.86 (B) \\
\hline 17 & 4-OH-3-R-1-naphthyl ${ }^{b}$ & 35 & $232-232.5$ & $0.28(\mathrm{C})$ \\
\hline 18 & 2-naphthyl & 93 & $232-232.5(232[45])$ & $0.71(\mathrm{~A})$ \\
\hline 19 & 4-Ph- $\mathrm{C}_{6} \mathrm{H}_{4}$ & 64 & $243.5-244(230-245[46])$ & $0.68(\mathrm{~A})$ \\
\hline 20 & 2-fluoryl & 97 & $260-261$ & $0.71(\mathrm{~A})$ \\
\hline 21 & 9-phenanthryl & 82 & $232-232.5(244$ [47]) & $0.70(\mathrm{~A})$ \\
\hline 22 & 1-pyrenyl & 94 & 299 (299 [39]) & $0.81(\mathrm{~A})$ \\
\hline
\end{tabular}

${ }^{\mathrm{a}}$ Mobile phase (MP), A: $\mathrm{CH}_{2} \mathrm{Cl}_{2}, \mathrm{~B}: \mathrm{CH}_{2} \mathrm{Cl}_{2}: \mathrm{MeOH}$ 90:10, C: EtOAc:Et ${ }_{3} \mathrm{~N}$ 100:1; ${ }^{\mathrm{b}}$ Depicted on Scheme 2.

\subsubsection{Antibacterial Activity}

The antibacterial activities of aldazines 1-22 were evaluated against the Gram (+) strains Bacillus subtilis ATCC 6633, Bacillus idosus B241, Bacillus megaterium NRRL 1353895, Bacillus mycoides DSMZ 274, Bacillus cereus ATCC 11778, Acinetobacter johnstonii ATCC 17909, Staphylococcus aureus NRRL B 313, Sarcina lutea ATCC 9341, and Micrococcus luteus ATCC 9631, and the Gram (-) strain Escherichia coli ATCC 8739. Streptomycin and Gentamycin were used as standard drugs.

The antibacterial screening demonstrated that half of the tested products, listed on Table 2, are active against all strains. Inside the benzaldazine series 1-13, the unsubstituted compound 1 exhibited good activities, while the introduction of substituents in the phenyl ring led to loss of activity in general. The only exceptions were 4-hydroxy compound 2 and 2-chloro-4-fluoro derivative 12 , both possessing lower activity than 1 . The compareson with their non-active position analogues, 2 vs 3-5 and 12 vs 13, could be an indication that hydroxyl group and fluorine atom at para-position slightly reduce the activity of the unsubstituted azine 1, while their effect if ortho- or meta-positioned is significant. Contrary, the introduction of a hydroxyl group in 1-naphthaldazine 14 led to better inhibition against the most part of the Gram (+)-bacteria of the ortho-substituted compound, 14 vs 16, and commensurable or reduced activities of para-hydroxy product, 14 vs 15 . The incorporation of a side-chain in 17, 
Table 2. Aldazines with antibacterial activity; zone of inhibition ${ }^{\mathrm{a}}$ in $\mathbf{~ m m}$.

\begin{tabular}{|c|c|c|c|c|c|c|c|c|c|c|}
\hline Azine & $\begin{array}{l}\text { Bacillus } \\
\text { subtilis }\end{array}$ & $\begin{array}{l}\text { Bacillus } \\
\text { idosus }\end{array}$ & $\begin{array}{l}\text { Bacillus } \\
\text { megat. }\end{array}$ & $\begin{array}{c}\text { Bacillus } \\
\text { mycoides }\end{array}$ & $\begin{array}{l}\text { Bacillus } \\
\text { cereus }\end{array}$ & $\begin{array}{c}\text { Acinetob. } \\
\text { johnst. }\end{array}$ & $\begin{array}{l}\text { Staph. } \\
\text { aureus }\end{array}$ & $\begin{array}{c}\text { Sarcina } \\
\text { lutea }\end{array}$ & $\begin{array}{l}\text { Microc. } \\
\text { luteus }\end{array}$ & E. coli \\
\hline $\mathbf{1}^{\mathrm{b}}$ & 20 & 21 & 20 & 16 & 17 & 24 & 18 & 20 & 19 & 30 \\
\hline $2^{b}$ & 10 & 18 & 17 & 15 & 26 & 17 & 17 & 17 & 16 & 20 \\
\hline $12^{\mathrm{b}}$ & - & 15 & 14 & 16 & 17 & - & 12 & 14 & 15 & 25 \\
\hline $14^{b}$ & 19 & 18 & 20 & 11 & 22 & 18 & 21 & 23 & 21 & 24 \\
\hline $15^{\mathrm{b}}$ & 13 & 19 & 12 & 18 & 16 & 16 & 14 & 14 & 14 & 25 \\
\hline $16^{\mathrm{c}}$ & 17 & 26 & 24 & 19 & 25 & 27 & 15 & 25 & 22 & 25 \\
\hline $17^{\mathrm{c}}$ & 11 & 12 & 11 & 10 & 12 & 11 & 12 & 11 & 12 & 12 \\
\hline $18^{\mathrm{c}}$ & 20 & 23 & 21 & 16 & 20 & 25 & 22 & 25 & 23 & 30 \\
\hline $19^{c}$ & 15 & 19 & 20 & 15 & 19 & 17 & 17 & 18 & 20 & 37 \\
\hline $20^{c}$ & 22 & 25 & 25 & 14 & 22 & 26 & 23 & 20 & 25 & 23 \\
\hline $22^{\mathrm{c}}$ & 15 & 19 & 14 & 15 & 19 & 13 & 12 & 18 & 21 & 33 \\
\hline $\operatorname{Ref} 1^{\mathrm{b}, \mathrm{e}}$ & 35 & 30 & 30 & 35 & 30 & 30 & 32 & 27 & 30 & 35 \\
\hline $\operatorname{Ref} 2^{\mathrm{d}, \mathrm{f}}$ & 30 & 35 & 36 & 32 & 29 & 39 & 35 & 34 & 38 & 31 \\
\hline
\end{tabular}

${ }^{\mathrm{a}}$ DMSO solutions with different concentrations; indicated for each compound: ${ }^{\mathrm{b}} 25 \mathrm{mg} / \mathrm{ml} ;{ }^{\mathrm{c}} 12.5 \mathrm{mg} / \mathrm{ml}$; ${ }^{\mathrm{d}} 20 \mathrm{mg} / \mathrm{ml}$; ${ }^{\mathrm{e}}$ Streptomycin; ${ }^{\mathrm{f}}$ Gentamycin.

where the hydroxyl proton is strongly hydrogen-bonded with piperidine nitrogen, resulted in an additional reduction of the activity, 15 vs 17.

\subsubsection{Antifungal Activity}

The antifungal activity of compounds 1-22 were examined against the yeast strains Candida tropicalis ATCC 20336 and Saccharomyces cerevisiae ATCC 9763, and the fungal strain Penicillium chrysogenum CECT 2802. Fluconazole and Itraconazole were used as standard drugs.

The qualitative experiments showed that only four products, three naphthaldazines $(14,15$ and 18) and a fluorenealdazine, listed on Table 3, exhibit significant antifungal activities. The rest of the aldazines gave commensurable zones of inhibition of maximum 10-12 $\mathrm{mm}$. A comparison between the active compounds (Table 3 ) shows that inside the naphthaldazine series, 2-naphtal- dazine 18 is remarkably more active than its position isomer 1-naphthaldazine 14 against Saccharomyces cer-evisiae and Penicillium chrysogenum, while both com- pounds possess the same activity towards Candida tropicalis. From the other side, the position of the hydroxy substituent in 1-naphthaldazine displayed a reversed effect in respect to antibacterial tests. The pres ence of a 4-hydroxyl substituent, 14 vs 15, led to slight increase of the activity towards the yeast strains tested and reduced activity against the fungal strain, while 2-hydroxy derivative 16 was not active in general.
Table 3. Aldazines with antifungal activity; zone of inhibittion $^{\mathrm{a}}$ in $\mathbf{m m}$.

\begin{tabular}{cccc}
\hline Compound & $\begin{array}{c}\text { Candida } \\
\text { tropicalis }\end{array}$ & $\begin{array}{c}\text { Saccharomyces } \\
\text { cerevisiae }\end{array}$ & $\begin{array}{c}\text { Penicillium } \\
\text { chrysogenum }\end{array}$ \\
\hline $\mathbf{1 4}^{\mathrm{b}}$ & 20 & 18 & 18 \\
$\mathbf{1 5}^{\mathrm{b}}$ & 24 & 25 & 15 \\
$\mathbf{1 8}^{\mathrm{c}}$ & 20 & 30 & 35 \\
$\mathbf{2 0}^{\mathrm{c}}$ & 21 & 23 & 20 \\
Fluconazol $^{\mathrm{b}}$ & 35 & - & - \\
Itraconazole $^{\mathrm{c}}$ & - & 30 & - \\
\hline
\end{tabular}

${ }^{\mathrm{a}}$ DMSO solutions with different concentrations; indicated for each compound: ${ }^{\mathrm{b}} 25 \mathrm{mg} / \mathrm{ml} ;{ }^{\mathrm{c}} 12.5 \mathrm{mg} / \mathrm{ml}$.

\section{Conclusions}

A series of 19 known and 3 new symmetrical acyclic aromatic aldazines were obtained. Their qualitative antimicrobial activities were evaluated against 10 bacterial and 3 fungal species. Eleven compounds exhibited good to moderate antibacterial activities, while only four bicyclic azines possessed significant antifungal activities. It was observed that the introduction of substituents in the phenyl ring led to loss of antibacterial activity in general, whereas none of the compounds showed significant antifungal activity. The position isomers 1-naphthaldazine and 2-naphthaldazine exhibited commensurable antibac- 
terial activities, while 2-naphtaldazine was significantly more active against Saccharomyces cerevisiae and Penicillium chrysogenum. The introduction of a hydroxyl group in 1-naphthaldazine led to better inhibition against the most part of the Gram (+)-bacteria of the ortho-substituted compound and similar or reduced activities of para-hydroxy product. Contrary, the presence of a 4-hydroxy group led to slight increase of the activity towards the yeast strains tested and reduced activity against the fungal strain, whereas 2-hydroxy derivative was not active in general.

\section{Experimental}

\subsection{General}

All reagents were purchased from Aldrich, Merck and Fluka and were used without any further purification. Fluka silica gel/TLC-cards 60778 with fluorescent indicator $254 \mathrm{~nm}$ were used for TLC chromatography and $\mathrm{R}_{\mathrm{f}}$-values determination. The high performance flash chromatography (HPFC) purifications were carried out on a Biotage HorizonTM system (Charlottesville, Virginia, USA) on silica gel. The melting points were determined in capillary tubes on SRS MPA100 OptiMelt (Sunnyvale, CA, USA) automated melting point system. The NMR spectra were recorded on a Bruker Avance DRX 250 and Bruker Avance II+ 600 (where indicated) spectrometers (Rheinstetten, Germany); the chemical shifts were quoted in ppm in $\delta$-values against tetramethylsilane (TMS) as an internal standard and the coupling constants were calculated in Hz. The chemical shifts are given with different decimals according to the accuracy (fid resolution) of the corresponding experiments. The assignment of the signals was achieved on the bases of the cross-peaks in the 2D experiments (COSY, NOESY, HSQC, HMBC). For simplicity, the carbon bearing the azine moiety is designated as $\mathrm{C}-1$ in all products except 18,20 and 21 , where the nomenclature of the corresponding fused carbocycles is followed. The aldazines 11 and 19-22 are not enough soluble to record carbon spectra at room temperature in a reasonable time-scale. The ESI mass-spectra were recorded on a DFS High Resolution Magnetic Sector MS, Thermo Scientific (Waltham, MA, USA).

The reaction yields, melting points, and $\mathrm{R}_{\mathrm{f}}$-values of the products are listed on Table $\mathbf{1}$.

\subsection{Preparation of Aldazines 1-16 and 18-22}

To a solution of aldehyde $(10 \mathrm{mmol})$ in $\mathrm{EtOH}(20 \mathrm{ml})$ and DMF (5 ml, only for the preparation of 20-22) hydrazine hydrate $(5 \mathrm{mmol})$ and then a drop of conc. $\mathrm{H}_{2} \mathrm{SO}_{4}$ were added and the mixture was stirred at room temperature for $1 \mathrm{~h}$. The residue formed was filtered off, washed with water and then with EtOH and was dried in air.

1,2-Dibenzylidenehydrazine (1). NMR $\left(\mathrm{CDCl}_{3}\right):{ }^{1} \mathrm{H}$ $7.436(\mathrm{~m}, 6 \mathrm{H}, \mathrm{CH}-3, \mathrm{CH}-4$ and $\mathrm{CH}-5), 7.837$ (dd, 4H, J 2.0, 7.8, $\mathrm{CH}-2$ and $\mathrm{CH}-6), 8.661(\mathrm{~s}, 2 \mathrm{H}, \mathrm{CH}=\mathrm{N}) ;{ }^{13} \mathrm{C}$ $128.83(\mathrm{CH}-2$ and $\mathrm{CH}-6), 129.39(\mathrm{CH}-3$ and $\mathrm{CH}-5)$, $131.83(\mathrm{CH}-4), 134.27\left(C_{\text {quat }}-1\right), 161.89(\mathrm{CH}=\mathrm{N})$.

1,2-Bis(4-hydroxybenzylidene)hydrazine (2). NMR $\left(\mathrm{CDCl}_{3}: \mathrm{DMSO}_{6} \mathrm{~d}_{6} 2: 1\right):{ }^{1} \mathrm{H} 6.79$ (d, 4H, J 8.6, CH-3 and $\mathrm{CH}-5), 7.58$ (d, 4H, J 8.6, $\mathrm{CH}-2$ and $\mathrm{CH}-6), 8.45$ (s, 2H, $\mathrm{CH}=\mathrm{N}), 9.69$ (bs, $2 \mathrm{H}, \mathrm{OH}$, exchangeable signal); ${ }^{13} \mathrm{C}$ $115.52(\mathrm{CH}-3$ and $\mathrm{CH}-5), 125.09\left(\mathrm{C}_{\text {quat }}-1\right), 129.70(\mathrm{CH}-2$ and $\mathrm{CH}-6), 159.85\left(\mathrm{C}_{\text {quat }}-4\right), 160.52(\mathrm{CH}=\mathrm{N})$.

1,2-Bis(3-hydroxybenzylidene)hydrazine (3). NMR $\left(\mathrm{CDCl}_{3}: \mathrm{DMSO}_{\mathrm{d}} \mathrm{5}\right.$ :1): ${ }^{1} \mathrm{H} 6.86(\mathrm{~m}, 2 \mathrm{H}, \mathrm{CH}-5), 7.17(\mathrm{~m}$, $4 \mathrm{H}, \mathrm{CH}-2$ and $\mathrm{CH}-6), 7.24(\mathrm{dd}, 2 \mathrm{H}, \mathrm{J} 1.2,2.7, \mathrm{CH}-2)$, $8.45(\mathrm{~s}, 2 \mathrm{H}, \mathrm{CH}=\mathrm{N}), 9.16(\mathrm{bs}, 2 \mathrm{H}, \mathrm{OH}$, exchangeable signal); ${ }^{13} \mathrm{C} 114.20(\mathrm{CH}-2), 118.26(\mathrm{CH}-4), 119.49$ (CH-6), 129.23 (CH-5), $134.70\left(C_{\text {quat }}-1\right), 157.25\left(C_{\text {quat }}-3\right)$, $161.15(\mathrm{CH}=\mathrm{N})$.

1,2-Bis(2-hydroxybenzylidene)hydrazine (4). NMR $\left(\mathrm{CDCl}_{3}: \mathrm{CF}_{3} \mathrm{COOH} 3: 1\right):{ }^{1} \mathrm{H} 7.18(\mathrm{~m}, 4 \mathrm{H}, \mathrm{CH}-3$ and CH-5), 7.57 (dd, 2H, J 1.6, 8.0, CH-6), 7.69 (ddd, 2H, J 1.6, 7.3, 8.7, CH-4), $8.98(\mathrm{~s}, 2 \mathrm{H}, \mathrm{CH}=\mathrm{N}), 11.69$ (bs, OH, overlapped with $\mathrm{COOH}$ of $\left.\mathrm{CF}_{3} \mathrm{COOH}\right) ;{ }^{13} \mathrm{C} 114.23$ $\left(C_{\text {quat }}-1\right), 118.03(\mathrm{CH}-3$ or $\mathrm{CH}-5), 122.69(\mathrm{CH}-3$ or $\mathrm{CH}-5), 136.06$ (CH-4 or $\mathrm{CH}-6), 139.94(\mathrm{CH}-4$ or $\mathrm{CH}-6)$, $160.49\left(C_{\text {quat }}-2\right), 164.58(C \mathrm{H}=\mathrm{N})$; NMR $\left(\mathrm{DMSO}_{-} \mathrm{d}_{6}\right):{ }^{1} \mathrm{H}$ $6.96(\mathrm{~m}, 4 \mathrm{H}, \mathrm{CH}-3$ and $\mathrm{CH}-5), 7.40$ (ddd, 2H, J 1.8, 7.2, 8.3, CH-4), 7.69 (dd, 2H, J 1.9, 8.3, CH-6), 9.00 (s, 2H, $\mathrm{CH}=\mathrm{N}), 11.11$ (bs, $\mathrm{OH}$, exchangeable signal).

1,2-Bis(2-hydroxy-3-methoxybenzylidene)hydrazine (5). $\mathrm{NMR}\left(\mathrm{CDCl}_{3}\right):{ }^{1} \mathrm{H} 3.93\left(\mathrm{~s}, 6 \mathrm{H}, \mathrm{OCH}_{3}\right), 6.96(\mathrm{~m}, 6 \mathrm{H}, \mathrm{CH})$, $8.70(\mathrm{~s}, 2 \mathrm{H}, \mathrm{CH}=\mathrm{N}), 11.57$ (bs, $2 \mathrm{H}, \mathrm{OH}$, exchangeable); ${ }^{13} \mathrm{C} 56.16\left(\mathrm{OCH}_{3}\right), 115.03(\mathrm{CH}-4), 117.29\left(\mathrm{C}_{\text {quat }}-1\right)$, 119.40 (CH-5), 124.01 (CH-6), 148.30 ( $\left.C_{\text {quat }}\right), 149.63$ $\left(C_{\text {quat }}\right), 164.80(\mathrm{CH}=\mathrm{N})$; NMR $\left(\mathrm{DMSO}-\mathrm{d}_{6}\right):{ }^{1} \mathrm{H} 3.83(\mathrm{~s}$, $\left.6 \mathrm{H}, \mathrm{OCH}_{3}\right), 6.91$ (t, 2H, J 7.9, CH-5), 7.13 (dd, 2H, J 1.4, 8.1, CH-4), 7.29 (dd, 2H, J 1.4, 7.9, CH-6), 8.98 (s, 2H, $\mathrm{CH}=\mathrm{N}), 10.87(\mathrm{~s}, 2 \mathrm{H}, \mathrm{OH})$; NMR $\left(\mathrm{DMSO}_{-} \mathrm{d}_{6}\right):{ }^{1} \mathrm{H} 3.833$ $\left(\mathrm{s}, 6 \mathrm{H}, \mathrm{OCH}_{3}\right), 6.910(\mathrm{t}, 2 \mathrm{H}, \mathrm{CH}-5), 7.127$ (dd, 2H, J $\left.1.4, \mathrm{~J}_{45} 8.1, \mathrm{CH}-4\right), 7.288$ (dd, $\left.2 \mathrm{H}, \mathrm{J}_{46} 1.4, \mathrm{~J}_{56} 7.9, \mathrm{CH}-6\right)$, $8.985(\mathrm{~s}, 2 \mathrm{H}, \mathrm{CH}=\mathrm{N}), 10.869$ (s, 2H, OH).

1,2-Bis(4-dimethylaminobenzylidene)hydrazine (6). NMR $\left(\mathrm{CDCl}_{3}\right):{ }^{1} \mathrm{H} 3.034$ (s, 12H, $\left.\mathrm{NCH}_{3}\right), 6.717$ (d, 4H, J 8.9, $\mathrm{CH}-3$ and $\mathrm{CH}-5), 7.699$ (d, 4H, J 8.9, $\mathrm{CH}-2$ and $\mathrm{CH}-6)$, $8.574(\mathrm{~s}, 2 \mathrm{H}, \mathrm{CH}=\mathrm{N}) ;{ }^{13} \mathrm{C} 40.16\left(\mathrm{NCH}_{3}\right), 111.67(\mathrm{CH}-3$ and $\mathrm{CH}-5), 128.18\left(C_{\text {quat }}-1\right), 129.85(\mathrm{CH}-2$ and $\mathrm{CH}-6)$, $152.07\left(C_{\text {quat }}-4\right), 160.77(\mathrm{CH}=\mathrm{N})$.

1,2-Bis(4-chlorobenzylidene)hydrazine (7). $\mathrm{NMR}\left(\mathrm{CDCl}_{3}\right)$ : ${ }^{1} \mathrm{H} 7.46$ (ddd, 4H, J 1.8, 2.3, 8.4, $\mathrm{CH}-3$ and $\mathrm{CH}-5$ ), 7.81 (ddd, 4H, J 1.8, 2.3, 8.4, $\mathrm{CH}-2$ and $\mathrm{CH}-6), 8.60$ (s, 2H, $\mathrm{CH}=\mathrm{N}) ;{ }^{13} \mathrm{C} 129.15(\mathrm{CH}-2$ and $\mathrm{CH}-6), 129.74(\mathrm{CH}-3$ 
and $\mathrm{CH}-5$ of $\mathrm{Ar}), 132.50\left(C_{\text {quat }}-1\right), 137.34\left(C_{\text {quat }}-4\right)$, $161.01(C \mathrm{H}=\mathrm{N})$.

1,2-Bis(3-chlorobenzylidene)hydrazine (8). NMR $\left(\mathrm{CDCl}_{3}\right)$ : ${ }^{1} \mathrm{H} 7.46(\mathrm{~m}, 4 \mathrm{H}, \mathrm{CH}-4$ and $\mathrm{CH}-5), 7.72(\mathrm{dt}, 2 \mathrm{H}, \mathrm{J}$ 1.6, 7.2, $\mathrm{CH}-6), 7.88$ (dd, 2H, J 1.6, 1.8, CH-2), 8.59 (s, 2H, CH= $\mathrm{N}) ;{ }^{13} \mathrm{C} 126.99(\mathrm{CH}-2$ or $\mathrm{CH}-6), 128.14(\mathrm{CH}-2$ or $\mathrm{CH}-6)$, 130.06 (CH-4 or $\mathrm{CH}-5), 131.29$ (CH-4 or $\mathrm{CH}-5), 135.00$ $\left(C_{\text {quat }}-1\right.$ or $\left.C_{\text {quat }}-3\right), 135.74\left(C_{\text {quat }}-1\right.$ or $\left.C_{\text {quat }}-3\right), 161.06$ $(\mathrm{CH}=\mathrm{N})$.

1,2-Bis(2-chlorobenzylidene)hydrazine (9). NMR $\left(\mathrm{CDCl}_{3}\right.$ : DMSO-d $\left._{6} 1: 3\right):{ }^{1} \mathrm{H} 7.43(\mathrm{~m}, 2 \mathrm{H}, \mathrm{CH}), 7.51(\mathrm{~m}, 4 \mathrm{H}, \mathrm{CH})$, $8.16(\mathrm{~m}, 2 \mathrm{H}, \mathrm{CH}), 8.97(\mathrm{~s}, 2 \mathrm{H}, \mathrm{CH}=\mathrm{N}) ;{ }^{13} \mathrm{C} 127.21$ (CH-5 or $\mathrm{CH}-6), 127.90$ (CH-5 or $\mathrm{CH}-6), 129.82(\mathrm{CH}-3)$, $130.47\left(C_{\text {quat }}-1\right), 132.59(\mathrm{CH}-4), 134.68\left(C_{\text {quat }}-2\right), 160.52$ $(\mathrm{CH}=\mathrm{N})$.

1,2-Bis(2,6-dichlorobenzylidene)hydrazine (10). NMR $\left(\mathrm{CDCl}_{3}\right):{ }^{1} \mathrm{H} 7.28$ (dd, 2H, J 7.0, 9.0, $\left.\mathrm{CH}-4\right), 7.411(\mathrm{~d}, 2 \mathrm{H}$, $\mathrm{J} 7.0, \mathrm{CH}-3$ or $\mathrm{CH}-5$, overlapped signals), $7.413(\mathrm{~d}, 2 \mathrm{H}, \mathrm{J}$ 9.0, $\mathrm{CH}-3$ or $\mathrm{CH}-5$, overlapped signals), $8.82(\mathrm{~s}, 2 \mathrm{H}, \mathrm{CH}=$ $\mathrm{N}) ;{ }^{13} \mathrm{C} 128.94(\mathrm{CH}-3$ and $\mathrm{CH}-5), 130.26\left(\mathrm{C}_{\text {quat }}-1\right)$, $130.96(\mathrm{CH}-4), 135.52\left(C_{\text {quat }}-2\right.$ and $\left.C_{\text {quat }}-6\right), 157.47(\mathrm{CH}=$ $\mathrm{N})$.

1,2-Bis(2,4-dichlorobenzylidene)hydrazine (11). NMR $\left(\mathrm{CDCl}_{3}:\right.$ DMSO-d 6 : $\left.\mathrm{CF}_{3} \mathrm{COOH} 3: 1: 0.05\right):{ }^{1} \mathrm{H} 7.23$ (dd, $2 \mathrm{H}$, J 2.0, 8.6, CH-5), 7.36 (d, 2H, J 2.0, CH-3), 8.04 (d, 2H, J 8.6, $\mathrm{CH}-6), 8.84(\mathrm{~s}, 2 \mathrm{H}, \mathrm{CH}=\mathrm{N})$.

1,2-Bis(2-chloro-4-fluorobenzylidene)hydrazine (12). NMR $\left(\mathrm{CDCl}_{3}\right)$ : ${ }^{1} \mathrm{H} 7.08$ (dddd, $2 \mathrm{H}, \mathrm{J}_{5, \mathrm{CH}=\mathrm{N}} 0.6, \mathrm{~J}_{3,5} 2.5$, $\left.\mathrm{J}_{5, \mathrm{~F}} 7.8, \mathrm{~J}_{5,6} 8.7, \mathrm{CH}-5\right), 7.19\left(\mathrm{dd}, 2 \mathrm{H}, \mathrm{J}_{3,5} 2.5, \mathrm{~J}_{5, \mathrm{~F}} 8.4\right.$, $\mathrm{CH}-3$ ), 8.24 (dd, 2H, $\left.\mathrm{J}_{6, \mathrm{~F}} 6.3, \mathrm{~J}_{5,6} 8.8, \mathrm{CH}-6\right), 9.01$ (d, 2H, $\left.\mathrm{J}_{5, \mathrm{CH}=\mathrm{N}} 0.6, \mathrm{CH}=\mathrm{N}\right) ;{ }^{13} \mathrm{C} 114.92\left(\mathrm{~d}, \mathrm{~J}_{5, \mathrm{~F}} 21.7, \mathrm{CH}-5\right)$, 117.34 (d, J $\left.\mathrm{J}_{3, \mathrm{~F}} 24.9, \mathrm{CH}-3\right), 127.89$ (d, J $\left.\mathrm{J}_{1, \mathrm{~F}} 3.6, C_{\text {quat }}-1\right)$, $129.94\left(\mathrm{~d}, \mathrm{~J}_{6, \mathrm{~F}}\right.$ 9.2, CH-6), 136.79 (d, $\left.\mathrm{J}_{2, \mathrm{~F}} 10.5, C_{\text {quat }}-2\right)$, $158.10(\mathrm{CH}=\mathrm{N}), 164.16\left(\mathrm{~d}, \mathrm{~J}_{4, \mathrm{~F}} 255.9, C_{\text {quat }}-4\right) ; \mathrm{MS}$ $\left(\mathrm{ESI}^{+}\right) \mathrm{m} / \mathrm{z} 313\left(\mathrm{M}^{+}\right), \mathrm{C}_{14} \mathrm{H}_{8} \mathrm{Cl}_{2} \mathrm{~F}_{2} \mathrm{~N}_{2}$.

1,2-Bis(3-fluorobenzylidene)hydrazine (13). NMR $\left(\mathrm{CDCl}_{3}\right)$ : ${ }^{1} \mathrm{H} 7.20$ (dddd, $2 \mathrm{H}, \mathrm{J}_{4,6}$ 1.1, $\mathrm{J}_{4,2}$ 2.6, $\mathrm{J}_{4,5} 8.3, \mathrm{~J}_{4, \mathrm{~F}}$ 9.3, $\mathrm{CH}-4$ ), 7.46 (ddd, $2 \mathrm{H}, \mathrm{J}_{5,6} 6.8, \mathrm{~J}_{4,5} 8.3, \mathrm{~J}_{5, \mathrm{~F}}$ 13.7, $\mathrm{CH}-5$ ), 7.57 (dt, 2H, J $\left.\mathrm{J}_{4,6} 1.1, \mathrm{~J}_{2,6} 1.4, \mathrm{~J}_{5,6} 6.8, \mathrm{CH}-6\right), 7.61(\mathrm{dd}, 2 \mathrm{H}$, $\left.\mathrm{J}_{2,6} 1.4, \mathrm{~J}_{2,4} 2.6, \mathrm{~J}_{2, \mathrm{~F}} 9.2, \mathrm{CH}-2\right), 8.61\left(\mathrm{~d}, 2 \mathrm{H}, \mathrm{J}_{\mathrm{CH}=\mathrm{N}, \mathrm{F}} 1.1\right.$, $\mathrm{CH}=\mathrm{N}) ;{ }^{13} \mathrm{C} 114.55\left(\mathrm{~d}, \mathrm{~J}_{2, \mathrm{~F}} 22.5, \mathrm{CH}-2\right), 118.31\left(\mathrm{~d}, \mathrm{~J}_{4, \mathrm{~F}}\right.$ 21.6, CH-4), 124.86 (d, J $\left.\mathrm{J}_{6 \mathrm{~F}} 2.9, \mathrm{CH}-6\right), 130.37$ (d, J $\mathrm{J}_{5, \mathrm{~F}} 8.1$, CH-5), 136.24 (d, $\left.\mathrm{J}_{1, \mathrm{~F}} 7.6, C_{\text {quat }}-1\right), 161.16\left(\mathrm{~d}, \mathrm{~J}_{\mathrm{CH}=\mathrm{N}, \mathrm{F}} 3.1\right.$, $\mathrm{CH}=\mathrm{N}), 163.02\left(\mathrm{~d}, \mathrm{~J}_{3, \mathrm{~F}} 246.9, C_{\text {quat }}-3\right)$.

1,2-Bis(naphthalen-1-ylmethylene)hydrazine (14). NMR (600 MHz, DMSO-d $\left._{6}\right):{ }^{1} \mathrm{H} 7.661$ (ddd, $2 \mathrm{H}, \mathrm{J}_{79} 1.1, \mathrm{~J}_{78} 6.8$, $\mathrm{J}_{67}$ 8.0, $\mathrm{CH}-7$ ), 7.691 (dd, $2 \mathrm{H}, \mathrm{J}_{23} 7.3, \mathrm{~J}_{34} 8.1, \mathrm{CH}-3$ ), 7.725 (ddd, 2H, $\mathrm{J}_{68} 1.3, \mathrm{~J}_{78} 6.8, \mathrm{~J}_{89} 8.4, \mathrm{CH}-8$ ), 8.076 (d, $\left.2 \mathrm{H}, \mathrm{J}_{67} 8.0, \mathrm{CH}-6\right), 8.147$ (d, 2H, $\left.\mathrm{J}_{34} 8.1, \mathrm{CH}-4\right), 8.194$ (dd, 2H, J $\mathrm{J}_{24}$ 0.7, $\mathrm{J}_{23} 7.3, \mathrm{CH}-2$ ), 9.212 (d, 2H, J $\mathrm{J}_{89} 8.4$, $\mathrm{CH}-9), 9.459(\mathrm{~s}, 2 \mathrm{H}, \mathrm{CH}=\mathrm{N}) ;{ }^{13} \mathrm{C} 125.09(\mathrm{CH}-9), 125.56$ (CH-3), 126.46 (CH-7), 127.62 (CH-8), 128.79 (CH-6), $129.17\left(C_{\text {quat }}-5\right), 130.42(\mathrm{CH}-2), 130.63\left(C_{\text {quat }}-10\right)$,
$131.96(\mathrm{CH}-4), 133.55\left(C_{\text {quat }}-1\right), 162.02(\mathrm{CH}=\mathrm{N})$.

1,2-Bis((4-hydroxynaphthalen-1-yl)methylene)hydrazi ne (15). NMR (DMSO- $\mathrm{d}_{6}$ ): ${ }^{1} \mathrm{H} 7.03$ (d, 2H, J 8.0, CH-3), 7.58 (ddd, 2H, J 1.1, 6.8, 8.2, CH-8), 7.70 (ddd, 2H, J 1.5, 6.8, 8.4, CH-7), 7. 97 (d, 2H, J 8.2, CH-2), 8.30 (dd, $2 \mathrm{H}$, J 1.1, 8.2, CH-9), $9.25(\mathrm{~s}, 2 \mathrm{H}, \mathrm{CH}=\mathrm{N}), 9.30(\mathrm{~d}, 2 \mathrm{H}, \mathrm{J} 8.4$, $\mathrm{CH}-6), 10.96$ (bs, $\mathrm{OH}) ;{ }^{13} \mathrm{C} 108.54(\mathrm{CH}-3), 120.87$ $\left(C_{\text {quat }}-1\right), 123.11(\mathrm{CH}-9), 125.25\left(C_{\text {quat }}-5\right), 125.64(\mathrm{CH}-6)$, $125.82(\mathrm{CH}-8), 128.33(\mathrm{CH}-7), 132.67\left(C_{\text {quat }}-10\right), 133.38$ $(\mathrm{CH}-2), 157.16\left(C_{\text {quat }}-4\right), 162.00(\mathrm{CH}=\mathrm{N})$.

1,2-Bis((2-hydroxynaphthalen-1-yl)methylene)hydrazi ne (16). NMR $\left(\mathrm{CDCl}_{3}: \mathrm{CF}_{3} \mathrm{COOH} 3: 1\right):{ }^{1} \mathrm{H} 7.30(\mathrm{~d}, 2 \mathrm{H}, \mathrm{J}$ 9.0, $\mathrm{CH}-3$ ), 7.50 (dd, 2H, J 7.1, 8.1, $\mathrm{CH}-7$ ), 7.68 (ddd, 2H, J 1.2, 7.1, 8.5, CH-6), 7.81 (d, 2H, J 8.1, CH-8), 8.07 (d, 2H, J 9.0, CH-4), 8.23 (d, 2H, J 8.5, CH-5), 9.70 (s, $2 \mathrm{H}, \mathrm{CH}=\mathrm{N}$ ), 11.08 (bs, $\mathrm{OH}$, overlapped with $\mathrm{COOH}$ of $\left.\mathrm{CF}_{3} \mathrm{COOH}\right) ;{ }^{13} \mathrm{C} 106.43\left(C_{\text {quat }}-1\right), 118.08(\mathrm{CH}), 120.11$ $(\mathrm{CH}), 126.13(\mathrm{CH}), 128.98\left(C_{\text {quat }}\right), 130.27(\mathrm{CH}), 130.69$ $(\mathrm{CH}), 132.79\left(\mathrm{C}_{\text {quat }}\right), 141.76(\mathrm{CH}), 157.90(\mathrm{CH}=\mathrm{N})$, $163.29\left(C_{\text {quat }}-2\right)$.

1,2-Bis(naphthalen-2-ylmethylene)hydrazine (18). NMR $\left(600 \mathrm{MHz}, \mathrm{DMSO}-\mathrm{d}_{6}, 70^{\circ} \mathrm{C}\right):{ }^{1} \mathrm{H} 7.634(\mathrm{~m}, 4 \mathrm{H}, \mathrm{CH}-7$ and $\mathrm{CH}-8), 8.012$ (d, 2H, J $\left.{ }_{67} 7.2, \mathrm{CH}-6\right), 8.054(\mathrm{~m}, 4 \mathrm{H}, \mathrm{CH}-4$ and $\mathrm{CH}-9$ ), $8.162\left(\mathrm{dd}, 2 \mathrm{H}, \mathrm{J}_{13} 1.3, \mathrm{~J}_{34} 8.5, \mathrm{CH}-3\right), 8.395$ (s, 2H, CH-1), $8.938(\mathrm{~s}, 2 \mathrm{H}, \mathrm{CH}=\mathrm{N}) ;{ }^{13} \mathrm{C} 123.99(\mathrm{CH}-3)$, 127.31 (CH-6), 128.10 (CH-7), 128.29 (CH-8), 129.00 (CH-9), $129.08(\mathrm{CH}-4), 131.04(\mathrm{CH}-1), 132.15\left(\mathrm{C}_{\text {quat }}-5\right)$, $133.33\left(C_{\text {quat }}-10\right), 134.93\left(C_{\text {quat }}-2\right), 161.48(\mathrm{CH}=\mathrm{N})$.

1,2-Bis(biphenyl-4-ylmethylene)hydrazine (19). NMR $\left(\mathrm{CDCl}_{3}:\right.$ DMSO- $\mathrm{d}_{6}: \mathrm{CF}_{3} \mathrm{COOH}$ 1:1:0.1): ${ }^{1} \mathrm{H} 7.33(\mathrm{~m}, 6 \mathrm{H}$, $\mathrm{CH}), 7.54(\mathrm{dt}, 4 \mathrm{H}, \mathrm{J} 1.5,7.0, \mathrm{CH}), 7.60$ (dt, 4H, J 1.7, 8.4, $\mathrm{CH}), 7.82(\mathrm{dt}, 4 \mathrm{H}, \mathrm{J} 1.7,8.4, \mathrm{CH}), 8.59(\mathrm{~s}, 2 \mathrm{H}, \mathrm{CH}=\mathrm{N})$.

1,2-Bis((9H-fluoren-2-yl)methylene)hydrazine (20). NMR $\left(600 \mathrm{MHz}, \mathrm{DMSO}-\mathrm{d}_{6}, 70^{\circ} \mathrm{C}\right):{ }^{1} \mathrm{H} 4.035\left(\mathrm{~s}, 4 \mathrm{H}, \mathrm{CH}_{2}\right)$, 7.391 (td, 2H, J 1.2, 7.3, CH-7), 7.440 (td, 2H, J 1.0, 7.3, CH-6), 7.647 (dt, 2H, J 0.9, 7.3, CH-8), 7.928 (dd, 2H, J 0.9, 7.9, CH-3), 7.974 (dd, 2H, J 1.0, 7.3, CH-5), 8.021 (d, 2H, J 7.9, CH-4), 8.126 (s, 2H, CH-1), 8.782 (s, 2H, $\mathrm{CH}=\mathrm{N}) ; \mathrm{MS}\left(\mathrm{ESI}^{+}\right) \mathrm{m} / \mathrm{z} 385\left(\mathrm{M}^{+}\right), \mathrm{C}_{28} \mathrm{H}_{20} \mathrm{~N}_{2}$.

1,2-Bis(phenanthren-9-ylmethylene)hydrazine (21). NMR (DMSO-d ${ }_{6}$ ): ${ }^{1} \mathrm{H} 7.82(\mathrm{~m}, 4 \mathrm{H}, \mathrm{CH}), 8.17$ (d, 2H, J 7.4, $\mathrm{CH}$ ), 8.57 (s, 2H, CH-10), 8.93 (d, 2H, J 7.7, CH), 8.99 $(\mathrm{m}, 2 \mathrm{H}, \mathrm{CH}), 9.38(\mathrm{~m}, 2 \mathrm{H}, \mathrm{CH}), 9.50(\mathrm{~s}, 2 \mathrm{H}, \mathrm{CH}=\mathrm{N})$.

1,2-Bis(pyren-1-ylmethylene)hydrazine (22). NMR (600 MHz, DMSO-d $\mathrm{d}_{6}$ ): ${ }^{1} \mathrm{H} 8.076$ (t, 2H, J 7.6, CH-7), 8.161 (m, 4H, CH), $8.245(\mathrm{~m}, 4 \mathrm{H}, \mathrm{CH}), 8.291(\mathrm{~m}, 4 \mathrm{H}, \mathrm{CH})$, 8.396 (d, 2H, J 8.1, CH), 8.708 (d, 2H, J 9.4, CH), 8.782 $(\mathrm{s}, 2 \mathrm{H}, \mathrm{CH}=\mathrm{N})$.

\subsection{Preparation of \\ 1,2-bis((4-hydroxy-3-(morpholinomethyl) naphthalen-1-yl)methylene)hydrazine (17)}

To a solution of $15(1 \mathrm{mmol})$ in benzene $(20 \mathrm{ml})$ mor- 
pholine $(2.1 \mathrm{mmol})$, paraformaldehyde $(2.2 \mathrm{mmol})$, and $p$-toluenesulfonic acid $(10 \mathrm{mg})$ were added and the mixture was refluxed with stirring for $4 \mathrm{~h}$. The products were partitioned between aq. $\mathrm{K}_{2} \mathrm{CO}_{3}$ and $\mathrm{CH}_{2} \mathrm{Cl}_{2}$. The organic layer was washed with water, dried over $\mathrm{Na}_{2} \mathrm{SO}_{4}$, evaporated to dryness, and purified by HPFC on silica gel; mobile phase with a gradient of polarity from hexane:EtOAc: $\mathrm{Et}_{3} \mathrm{~N}$ 50:50:0.5 to EtOAc: $\mathrm{Et}_{3} \mathrm{~N}$ 100:1; NMR (DMSO-d ${ }_{6}: \mathrm{CF}_{3} \mathrm{COOH}$ 5:1): ${ }^{1} \mathrm{H} 3.264$ (bm, 4H, $\mathrm{CH}_{2}-\mathrm{N}$ morpholine), 3.387 (bm, 4H, $\mathrm{CH}_{2}-\mathrm{N}$ morpholine), 3.706 (bm, 4H, $\mathrm{CH}_{2}-\mathrm{O}$ morpholine), $3.922\left(\mathrm{bm}, 4 \mathrm{H}, \mathrm{CH}_{2}-\mathrm{O}\right.$ morpholine), $4.583\left(\mathrm{~s}, 4 \mathrm{H}, \mathrm{Ar}-\mathrm{CH}_{2}-\mathrm{N}\right), 7.604$ (ddd, $2 \mathrm{H}, \mathrm{J}$ 1.1, 6.9, 8.4, $\mathrm{CH}-7$ or $\mathrm{CH}-8$ ), 7.703 (ddd, 2H, J 1.3, 6.9, $8.5, \mathrm{CH}-7$ or $\mathrm{CH}-8), 8.121$ (s, $2 \mathrm{H}, \mathrm{CH}-2), 8.467$ (dd, $2 \mathrm{H}$, J 1.1, 8.5, CH-6 or CH-9), 9.132 (dd, 2H, J 1.2, 8.4, $\mathrm{CH}-6$ or $\mathrm{CH}-9), 9.273(\mathrm{~s}, 2 \mathrm{H}, \mathrm{CH}=\mathrm{N}), 9.928(\mathrm{bs}, 2 \mathrm{H}$, $\mathrm{OH}$, exchangeable signal); ${ }^{13} \mathrm{C} 52.04\left(\mathrm{CH}_{2}-\mathrm{N}\right.$ morpholine), $55.76\left(\mathrm{Ar}-\mathrm{CH}_{2}-\mathrm{N}\right), 64.06\left(\mathrm{CH}_{2}-\mathrm{O}\right.$ morpholine $)$, $110.83\left(C_{\text {quat }}\right), 121.96\left(C_{\text {quat }}\right), 124.25(\mathrm{CH}), 125.80(\mathrm{CH})$, $126.20\left(C_{\text {quat }}\right), 126.70(\mathrm{CH}), 129.51(\mathrm{CH}), 133.69$ ( $\left.C_{\text {quat }}\right)$, $135.59(\mathrm{CH}), 157.46\left(C_{\text {quat }}-4\right), 161.50(\mathrm{CH}=\mathrm{N})$; $\mathrm{MS}$ $\left(\mathrm{ESI}^{+}\right) \mathrm{m} / \mathrm{z} 539\left(\mathrm{M}^{+}\right), \mathrm{C}_{32} \mathrm{H}_{34} \mathrm{~N}_{4} \mathrm{O}_{4}$.

\section{Acknowledgements}

The financial support by the National Research Fund of Bulgaria, Projects TK-X-1716 and UNA-17/2005, is gratefully acknowledged.

\section{REFERENCES}

[1] D. Kolbah and D. Korunčev, "Methoden zur Herstellung und Umwandlung von Azinen," In: Methoden der Organischen Chemie (Houben-Weyl), Vol. 10, part 2, Georg Thieme Verlag, Stuttgart, 1967, pp. 85-122.

[2] S. Dayagi and Y. Degani, "Formation of the Carbon-Nitrogen Double Bond," In: S. Patai, Ed., The Chemistry of the Carbon-Nitrogen Double Bonds, Interscience, New York, 1970, pp. 61-147. doi:10.1002/9780470771204.ch2

[3] W. Borsche, "Eine neue Reaction der Semicarbazone," Berichte der Deutschen Chemischen Gesellschaft, Vol. 34, No. 3, October-December 1901, pp. 4297-4302. doi:10.1002/cber.190103403161

[4] S. N. Shah and N. K. Chudgar, "Thermolysis of Semicarbazones to the Corresponding Azines Through Reactive $\mathrm{N}$-Substituted Isocyanate Intermediates," Molecules, Vol. 5, No. 4, April 2000, pp. 657-664. doi: $10.3390 / 50400657$

[5] H. Loghmani-Khouzani, M. M. M. Sadeghi, J. Safari and M. S. Abdorrezaie, "A Convenient Synthesis of Azines under Solvent-Free Conditions Using Microwave Irradiation," Journal of Chemical Research (S), No. 2, February 2001, pp. 80-81.

[6] S. P. Simeonov, V. B. Kurteva and R. P. Bontchev, "One-Pot Solvent-Free Synthesis of Symmetrical Azines under Microwave Irradiation," Bulgarian Chemical Communications, Vol. 40, No. 4, 2008, pp. 409-417.

[7] W. J. Haggerty and C. C. Cheng, "Antitumor Activity of Some Azine and Hydrazone Derivatives of 1,4-Dimethoxy-2-Butanone," Journal of Medicinal Chemistry, Vol. 13, No. 3, May 1970, pp. 574-575. doi:10.1021/jm00297a067

[8] J. R. Dimmock, P. Kumar, J. W. Quail, U. Pugazhenthi, J. Yang, M. Chen, R. S. Reid, T. M. Allen, G. Y. Kao, S. P. C. Cole, G. Batist, J. Balzarini and E. De Clercq, "Synthesis and Cytotoxic Evaluation of Some Styryl Ketones and Related Compounds," European Journal of Medicinal Chemistry, Vol. 30, No. 3, 1995, pp. 209-217.

[9] A. I. Khodair and P. Bertrand, "A New Approach to the Synthesis of Substituted 4-Imidazolidinones as Potential Antiviral and Antitumor Agents," Tetrahedron, Vol. 54, No. 19, May 1998, pp. 4859-4872. doi:10.1016/S0040-4020(98)00170-7

[10] H. I. Gul, M. Gul, J. Vepsälainen, E. Erciyas and O. Hänninen, "Cytotoxicity of Some Azines of Acetophenone Derived Mono-Mannich Bases against Jurkat Cells," Biological and Pharmaceutical Bulletin, Vol. 26, No. 5, May 2003, pp. 631-637. doi:10.1248/bpb.26.631

[11] H. N. Pati, U. Das, R. K. Sharma and J. R. Dimmock, "Cytotoxic Thiol Alkylators," Mini Reviews in Medicinal Chemistry, Vol. 7, No. 2, February 2007, pp. 131-139. doi: $10.2174 / 138955707779802642$

[12] N. Haider, T. Kabicher, J. Käferböck and A. Plenk, "Synthesis and In-vitro Antitumor Activity of 1-[3(Indol-1-yl) prop-1-yn-1-yl] phthalazines and Related Compounds," Molecules, Vol. 12, No. 8, August 2007, pp. 1900-1909. doi:10.3390/12081900

[13] Y. Sawa and M. Hoten, "Antibacterial Activity of Basic Dyes on the Dyed Acrylic Fibers," Sen'i Gakkaishi, Vol. 57, No. 5, May 2001, pp. 153-158. doi:10.2115/fiber.57.153

[14] R. N. Asolkar, V. P. Kamat, I. Wagner-Döbler and H. Laatsch, "Limnazine, the First Bacterial Azine Derivative from Bacillus sp. GW90a," Journal of Natural Products, Vol. 65, No. 11, Novembre 2002, pp. 1664-1666. doi:10.1021/np020108n

[15] I. A. Danish and K. R. Prasad, "Synthesis and Characterisation of $N, N$ '-Biscarbazolyl Azine and $N, N$ '-Carbazolyl Hydrazine Derivatives and Their Antimicrobial Studies," Acta Pharmaceutica, Vol. 54, No. 2, June 2004, pp. 133-142.

[16] H. I. Gul, F. Sahin, M. Gul, S. Ozturk and K. O. Yerdelen, "Evaluation of Antimicrobial Activities of Several Mannich Bases and Their Derivatives," Archiv der Pharmazie, Vol. 338, No. 7, July 2005, pp. 335-338. doi:10.1002/ardp.200400962

[17] M. N. Kumaraswamy and V. P. Vaidya, "Novel Method for the Synthesis of Symmetrical and Asymmetrical Azines Involving Naphtho [2,1-b] Furan and Their Antimicrobial Activity," Indian Journal of Heterocyclic Chemistry, vol. 14, no. 3, January-March 2005, pp. 193-196.

[18] J. Jayabharathi, A. Thangamani, M. Padmavathy and B. 
Krishnakumar, "Synthesis and Microbial Evaluation of Novel N(1)-Arilidene-N(2)-t(3)-methyl-r(2), c(6)-diarylpiperidin-4-one Azine Derivatives," Medicinal Chemistry Research, Vol. 15, No. 7-8, August 2007, pp. 431-442. doi:10.1007/s00044-006-0014-0

[19] J. Jayabharathi, V. Thanikachalam, A. Thangamani and M. Padmavathy, "Synthesis, $\mathrm{AM}_{1}$ Calculation, and Biological Studies of Thiopyran-4-one and Their Azine Derivatives," Medicinal Chemistry Research, Vol. 16, No. 6, December 2007, pp. 266-279. doi:10.1007/s00044-007-9029-4

[20] G. Aridoss, S. Amirthaganesan, M. S. Kim, J. T. Kim and Y. T. Jeong, "Synthesis, Spectral and Biological Evaluation of Some New Thiazolidinones and Thiazoles Based on t-3-Alkyl-r-2,c-6-diarylpiperidin-4-ones," European Journal of Medicinal Chemistry, Vol. 44, No. 10, October 2009, pp. 4199-4210. doi:10.1016/j.ejmech.2009.05.015

[21] R. W. Lange, "BXT-51072 OXIS International," Current Opinion in Anti-inflammatory and Immunomodulatory Investigational Drugs, Vol. 2, No. 4, 2000, pp. 338-341.

[22] J. L. Vennerstrom, M. T. Makler, C. K. Angerhofer and J. A. Williams, "Antimalarial Dyes Revisited-Xanthenes, Azines, Oxazines, and Thiazines," Antimicrobial Agents and Chemotherapy, vol. 39, no. 12, December 1995, pp. 2671-2677.

[23] F. D. Popp, "Potential Anticonvulsants .8. Some Hydrazones of Indole-3-Carboxaldehyde," Journal of Heterocyclic Chemistry, Vol. 21, No. 2, 1984, pp. 617-619. doi:10.1002/jhet.5570210273

[24] H. I. Gul, U. Calis and J. Vepsalainen, "Synthesis of Some Mono-Mannich Bases and Corresponding Azine Derivatives and Evaluation of Their Anticonvulsant Activity," Arzneimittel-Forschung-Drug Research, Vol. 54, No. 7, 2004, pp. 359-364.

[25] M. Eberle, S. Farooq, A. Jeanguenat, D. Mousset, A. Steiger, S. Trah, W. Zambach and A. Rindlisbacher, "Azine Derivatives as a New Generation of Insect Growth Regulators," Chimia, Vol. 57, No. 11, 2003, pp. 705-709.

[26] I. Picón-Ferrer, F. Hueso-Ureña, N. A. Illán-Cabeza, S. B. Jiménez-Pulido, J. M. Martínez-Martos, M. J. RamírezExpósito and M. N. Moreno-Carretero, "Chloro-fac-tricarbonylrhenium(I) Complexes of Asymmetric Azines Derived from 6-Acetyl-1,3,7-trimethylpteridine-2,4(1h,3h)dione with Hydrazine and Aromatic Aldehydes: Preparation, Structural Characterization and Biological Activity Against Several Human Tumor Cell Lines," Journal of Inorganic Biochemistry, Vol. 103, No. 1, January 2009, pp. 94-100.

[27] M. Revanasiddappa, T. Suresh, S. Khasim, S. C. Raghavendray, C. Basavaraja and S. D. Angadi, "Electrical Conductivity Studies on $\mathrm{Co}(\mathrm{II}), \mathrm{Cu}(\mathrm{II}), \mathrm{Ni}(\mathrm{II})$ and Cd(II) Complexes of Azines," E-Journal of Chemistry, Vol. 5, No. 4, 2008, pp. 395-403.

[28] G. Gürkök, N. Altanlar and S. Suzen, "Investigation of Antimicrobial Activities of Indole-3-Aldehyde Hydrazide/Hydrazone Derivatives," Chemotherapy, Vol. 55, No. 1, 2009, pp. 15-19.
[29] G. Gürkök, T. Coban and S. Suzen, "Melatonin Analogue New Indole Hydrazide/Hydrazone Derivatives with Antioxidant Behavior: Synthesis and Structure-Activity Relationships," Journal of Enzyme Inhibition and Medicinal Chemistry, Vol. 24, No. 2, April 2009, pp. 506-515.

[30] J. R. Dimmock, K. K. Sidhu, S. D. Tumber, S. K. Basran, M. Chen, J. W. Quail, J. Yang, I. Rozas and D. F. Weaver, "Some Aryl Semicarbazones Possessing Anticonvulsant Activitie," European Journal of Medicinal Chemistry, Vol. 30, No. 4, 1995, pp. 287-301.

[31] J. A. O'Brien, W. Lemaire, T.-B. Chen, R. S. L. Chang, M. A. Jacobson, S. N. Ha, C. W. Lindsley, H. J. Schaffhauser, C. Sur, D. J. Pettibone, P. J. Conn and D. L. Williams Jr, "A Family of Highly Selective Allosteric Modulators of the Metabotropic Glutamate Receptor Subtype 5," Molecular Pharmacology, Vol. 64, No. 3, September 2003, pp. 731-740.

[32] L. Horner, W. Kirmse and H. Fernekess, "Azinmonoxyde, Darstellung und Eigenschaften," Chemische Berichte, Vol. 94, No. 1, Januar 1961, pp. 279-290.

[33] D. Vorländer, "Ueber krystallinisch-flüssige Substanzen," Berichte der deutschen chemischen Gesellschaft, Vol. 39, No. 1, Januar-Februar 1906, pp. 803-810.

[34] H. Franzen and T. Eichler, "Zur Kenntnis der Benzalhydrazine," Journal für Praktische Chemie, Vol. 82, No. 1, December 1910, pp. 241-251.

[35] A. P. Terent'ev, E. G. Rukhadze, G. P. Talyzenkova and G. V. Panova, "Azomethines of $o$-vanilin and their chelate compounds," Zhurnal Obshchei Khimii, Vol. 36, No. 9, 1966, pp. 1590-1594.

[36] G. F. D'alelio, J. V. Crivello, R. K. Schoenig and T. F. Huemmer, "Polymeric Schiff Bases. II: The Synthesis of Azomethines by a Continuous Azeotropic Method," Journal of Macromolecular Science, Part A Pure and Applied Chemistry, Vol. 1, No. 7, 1967, pp. 1251-1258.

[37] T. Curtius, F. Mayer, R. Korte and H. Wewer, "Über die Reduktion der aromatischen Aldazine," Journal für Praktische Chemie, vol. 85, no. 1, Mai 1912, pp. 137-188.

[38] T. Curtius and H. Pauli, "Oxydation von symmetrischen secundären Benzylhydrazinen, R. $\mathrm{CH}_{2} \cdot \mathrm{NH} . \mathrm{NH} . \mathrm{CH}_{2} \cdot \mathrm{R}$, zu Hydrazonen, R.CH:N.NH.CH 2. R," Berichte der deutschen chemischen Gesellschaft, Vol. 34, No.1, Januar-April 1901, pp. 847-853.

[39] G. Lock and K. Stach, "Über die katalytische Zersetzung der Hydrazone, I. Mitteil.: Aromatische Aldehydhydrazone," Berichte der deutschen chemischen Gesellschaft, Vol. 76, No. 12, Dezember 1943, pp. 1252-1256.

[40] N. P. Buu-Hoï and G. Saint-Ruf, "Synthèse des diaryl-1,2 éthylènes par thermolyse des aldazines en milieu liquide," Bulletin de la Société Chimique de France, No. 6, 1968, pp. 2489-2492.

[41] N. P. Buu-Hoï and G. Saint-Ruf, "La Thermodécomposition des Aldazines Aromatiques et ses Applications à la Chimie Préparative," Bulletin de la Société Chimique de France, No. 3, 1967, pp. 955-960.

[42] T. Curtius, R. Glaser, G. Detoros, L. F. Potter, K. Traumann, L. F. Guttmann, J. Schmittmann and H. Pauli, 
"Über die Reduktion der aromatischen Aldazine. Reduktion von o- und m-Oxybenzaldazin in alkalischer Lösung," Journal für Praktische Chemie, Vol. 85, No. 1, Mai 1912, pp. 393-484.

[43] L. Gattermann, "Synthesen Aromatischer Aldehyde," Justus Liebigs Annalen der Chemie, Vol. 357, No. 2-3, 1907, pp. 313-383.

[44] L. Gattermann and T. von Horlacher, "Synthese von Oxyaldehyden der Naphtalinreihe," Berichte der deutschen chemischen Gesellschaft, Vol. 32, No. 1, Januar-April 1899, pp. 284-286.

[45] G. W. Monier-Williams, "Synthesis of Aldehydes by
Grignard's Reaction," Journal of the Chemical Society, Vol. 89, No. 1, 1906, pp. 273-280.

[46] L. Y. Malkes and A. I. Timchenko, "Synthesis of trans-n, n'-diphenylstilbene," Zhurnal Obshchei Khimii, Vol. 31, No. 2, 1961, pp. 560-562.

[47] G. Saint-Ruf, B. Kirkiacharian, N. P. Buu-Hoï and C. Mentzer, "Détection de la Formation de Radicaux Libres au Cours de la Thermolyse des Aldazines Aromatiques," Bulletin de la Société Chimique de France, No. 8, 1967, pp. 3078-3079.

[48] European Pharmacopoia, $3^{\text {th }}$ Edition, Deutscher Apotheker Verlag, Stuttgart, 1997, pp. 113-118. 